\title{
A Potential Application of Gale-Shapley Stable Marriage Model in the Babysitter-Households Matching in China
}

\author{
Luyin Wang
}

\author{
Guangdong Country Garden School \\ Corresponding author.Email: luyinwang115@gmail.com
}

\begin{abstract}
The traditional Gale-Shapley two-sided matching has been applied to high admission system in the US, which helps reduce the inefficiency when matching students and schools. In other areas like kidney exchange market, market design also plays an important role. Facing the upcoming third child policy in China, the author tries to apply the insights from market design to help better match the surging demand and supply of babysitters in China. This paper uses the ideas from Gale-Shapley Stable Marriage Model to suggest an online matching system which focuses on the matching mechanism between households and babysitters. Then characteristics of the matching system are discussed to test if it is an ideal system. Significance of such system on both individual level and the greater economy are then examined. The limitations of the model and potential solution are brought up at the end before the final conclusion about this online matching system is drawn.
\end{abstract}

Keywords: Deferred-acceptance algorithm, babysitter matching, Care Industry, market design, two-sided matching

\section{INTRODUCTION}

After the implementation of 'Second Child Policy' in China in 2016, number of newborns increased [1], which drove up the demand for babysitters in the care industry. As reported by 58.com, the largest employment website in china, there was a $32.9 \%$ increase in the job seekers in care industry, and among them, the largest increase was brought by babysitters, up to $47.1 \%$ in August 2018 [2]. The surging demand and supply impose pressure on the market and sometimes distortions occurs. Ren believed that the care industry in China was still immature, and lack of regulations and proper matching systems results in disbelieve of consumers [3]. To better match the two sides in this scenario, a properly designed matching system is needed. Therefore, the author suggests designing a new online matching system called ORSB which gains insights from Gale-Shapley Stable Marriage Model to better match the increasing demand and supply for babysitters in China, hence encouraging birthing to cope with aging problem in Chinese economy.

\section{INTRODUCTION TO RELATED CONCEPTS}

The traditional Gale-Shapley Stable Marriage Model assumes there is a finite set of men $m$ and a finite set of women $\mathrm{w}$, and the matching runs a Deferred-acceptance algorithm to match a man $\mathrm{m} 1$ to a woman w1 [4]. The stable matching reached is called $\mu 1$. Here, stable means no blocking individual or blocking pair. Blocking individual refers to someone who finds his or her current matching less preferred than another option, be it another matching partner or being unemployed (i.e., $\mathrm{m} 1$ prefers $\mathrm{w} 2$ than $\mathrm{w} 1 / \mathrm{m} 1$ ). Blocking pair occurs when a men-women pair find each other more preferable than their current partner. The rationale is that when blocking individual or blocking pair exist, they would 'divorce' with their partner, hence breaking the stability of current matching.

ORSB is the abbreviation of 'Online Recruitment System for Babysitter', and it adopts the main ideas from the Gale-Shapley Stable Marriage Model to construct a similar matching system which aims to meet the high demands for babysitters by helping households 
find preferable babysitters from a larger pool of unemployed babysitters who seek for employment.

\section{THE OPERATION OF ORSB}

There are generally 6 stages in ORSB: information input, preference input, selected jobs, ranking, proposing and final result release.

In the first part, two sides type in their basic information to the online system. For the babysitters, they would provide personal details including age, gender, approximate address, education level, qualifications obtained and previous working experience. For the households, they would add information about approximate home address, number of family members and age of the baby.

After completing personal information, two sides should input their preferences to help the centralized system to secondly filter out certain proportion of matchings. For both babysitters and households, they should provide the expected job responsibility (whether it is just taking care of the baby or involves cooking and washing as well), wage rate and working time (babysitters should specify their availability while households provide requirements for working hours per week). Please note that the preferences input here is not the final preferences used for matching but served as information collected for the centralized system to filter out those seemingly inappropriate matchings, such as long-distance babysitters and households. Hence, the matching system still adopts direct matching.

In the next stage, AI will analyze existing information and provide a list of vacancies that match babysitters' expectations. Each babysitter would receive such different list according to their own preferences and backgrounds. Then, babysitters would carefully compare the positions on the list and make comparisons. Finally, they come up with a preference ranking which places the most preferable job at the top. Inspired by 'Gale-Shapley Stable Marriage Problem Revisited: Strategic Issues and Applications' which suggests that requirement on a complete preference would reduce incentives to cheat [5], ORSB also has such requirement for both babysitters and households. Hence, it is not possible for either side to report certain options as 'unacceptable' in order to be better matched.

When babysitters successfully upload their preferences, the centralized system would run a Deferred-Acceptance algorithm. This means the online system would firstly propose to the top ranked households with information of babysitters provided and wait for the decision. If a household accepts the proposal, then the household and the babysitter are temporarily matched. If the first household rejects the proposal, then the system would make second proposal to the second ranked household. In the second round, the system would help those who were rejected at the first round to propose to their second choice. Again, if this household reports satisfied with the babysitter by accepting the proposal, the two sides would be temporarily matched. For households who receive two or more proposals, they may accept the most preferable and reject the rest. In the third and fourth round, proposals are made along the preference ranking and households always hold the most preferable proposal only. In this way, a babysitter would finally be matched with a household that he or she finds acceptable (i.e., The utility gained by being employed by that household is at least the same as being unemployed.).

\section{CHARACTERISTICS}

ORSB enjoys following characteristics which make it an ideal matching system, that is thickness, congestion-avoiding, stable, strategy proof, paretooptimality.

ORSB is designed as an official platform which is run by government staffs. Hence, it is comparatively more reliable compared to other private platforms for consumers. In the beginning, some coupons may be allocated to encourage registrations on the system. When more households have enjoyed the matching and correspondence services on the system, they are more likely to recommend it to others. Also, the system may cooperate with large skill schools, give free training for babysitters who are about to start working at the household matched in the system. Hence, more babysitters would also be attracted. According to Roth [6], this fulfills the characteristic of 'thickness'- 'to bring together a large enough population of buyers and sellers to produce satisfactory outcomes for both sides of a transaction.'

In some systems, congestion may be a side-effect of thickness. However, in ORSB, as the filtering and proposing process is actually done by computer, it speeds up the matching process and allows the system to work out a satisfactory matching for both babysitters and households as soon as possible.

The third characteristic is stable. As proofed by Gale and Shaley in their paper in 1962, there always exists a stable matching when running the DA algorithm. The situation is same in ORSB. As the proposals go down by the list, the final offer made to the babysitter must be the highest of those who is willing to give an offer. And as households are always holding the proposal of the most preferable babysitter, they do not have a better possible matching. In this way, both babysitters and households do not have the incentive to deviate from their current matching as there is not better option for them. Hence, the matching in ORSB is stable.

Secondly, the matching is strategy-proof for the proposer. The definition of strategy-proof is that 
everyone in the matching system finds that reporting their true preference weakly dominates telling lies (i.e., telling truths is at least as well as lying). Roth showed in his publishment 'The Economics of Matching: Stability and Incentive' that the man-proposing DA mechanism in the marriage model is strategyproof for the men, but not for the women [7]. Also, he further showed that no stable mechanism is strategyproof for all the agents. This is same in ORSB. As the proposal goes down the list, babysitters have the incentive to report their true preferences otherwise they may result in being matched with a less preferable household though a better option is available to them.

Another characteristic is 'Pareto optimality'. Gale and Shapley have worked to reach a men-optimal stable matching when they run a men-proposing algorithm [4]. The result could be easily changed to women-optimal stable matching if people use women-proposing algorithm. However, in 'Some Remarks on the Stable Matching Problem', Gale and Shapley discussed that women could still enjoy the women-optimal stable matching when men propose by reporting a false preference. To be more specific, they could report options below their women-optimal outcome as unacceptable, as if deleting them from their preference list [8]. In ORSB, the author tries to avoid such strategies by requiring both babysitters and households to submit a complete reference ranking (i.e., preventing behaviors like reporting 'unacceptable' options).

\section{SIGNIFICANCE}

Constructing an online matching system in the babysitter-households matching have multiple significance both in individual level and to the greater economy.

The system would help households to find reliable and suitable babysitters in a comparatively short time. Now, there is no centralized system to match the two sides, and most of the babysitter-households matching work is done by private agencies. However, without proper regulation on such agencies, there always lacks transparency when households choose babysitters. Rather than contacting directly to the babysitter, agencies meet with households and take advantage of the asymmetric information to exaggerate certain facts and fool the households. According to research done by Hebei Normal University on 500 babysitters, around $16 \%$ only received one-week training before taking the job, and only $24.2 \%$ of all interviewees have acquired qualification on caring [9]. At the end, new mothers always find it hard to find a preferable babysitter. Constructing ORSB would allow households to select and contact babysitters in an official platform with strict review on background information, thus making the selection process more reliable. The system also saves household a lot of time as the major 'proposing' process is done by computer.

Besides, the system is particularly beneficial to career women. A professional babysitter would make them less worried and concerned about child-caring, hence they would be able to return to career in a shorter time. Besides, when babies grow to children, babysitters could also help take care of them, so that mothers would not take leaves frequently from work. When female workers' working time is less affected by pregnancy and birthing, they are likely to suffer less form 'motherhood penalty' and enjoy higher chances for promotion. In this way, it would be expected that some women may enjoy higher wages, hence contributing to the narrowing of gender wage gap.

What's more, for babysitters, they now have a more professional access to find jobs and are able to meet more families. Without any mediator, babysitters would receive higher wages (previously certain proportion of their wage is given to the agency). This would allow employed babysitters to invest in retraining to gain higher qualification and accumulate experience. A higher wage also encourages more job seekers to join in the care industry. Thus, the increasing labor supply for babysitters would keep up with the surging number of newborns after the third-child policy.

The system also has macroeconomic significance. When baby-caring becomes easier and less timeconsuming for mothers, more childbearing age women may be encouraged to give birth, lifting up the birth rate in China. This fits Chinese government's aim of increasing birthing to cope with potential aging problem as more people would enter the labor force to bring more income home to the family to support the old in the long run [10].

Benefits could be observed in short run as well. Once the system becomes a major platform for household-babysitter matching with strict regulations. Government would find it much easier to collect taxation than before as every transaction on care services is listed clearly on the system now. Also, when the hiring process become standardized on the platform, there would exist less labor contract disputes, saving governments' human capitals on solving that.

Generally saying, constructing ORSB would allow babysitters and households to find preferable partner quickly while enabling career women to return to career with less concerns and then help to reduce negative impacts of birthing on women's career life. In addition, the economy may take advantage from easier tax collection, quicker population increase and more labor forces in the long run. 


\section{LIMITATIONS OF THE SYSTEM}

Though the above design sounds appealing, limitations exist. For example, mothers usually pay extra attention to the selection of babysitter, thus they are likely to require an interview before making an offer. However, interview requires a comparatively long time to conduct and adds more workload for the system to schedule an appropriate interview time. As the system aims to reduce the matching as much as possible, the author simplifies the scenario here and uses a video profile which would be compulsory when uploading personal information in the first step to replace traditional real interviews. Though this change would speed up the matching process, there is possibility that households find the babysitter unsatisfied when actually meet him or her, thus making the entire matching process effortless.

Apart from that, the author assumes the householdbabysitter matching is a one-to-one matching for simplification. However, in real life, cases in different households vary, some households may require more than one babysitter at a time. Thus, when actually designing the system, we need to make certain adaptation and personalization to it to better serve the users.

\section{CONCLUSION}

By applying the key ideas of Gale-Shapley Stable Marriage Model and DA algorithm, the author designed an online matching system named ORSB for babysitters and households. The market of babysitting is thick, and the system prevents it from being congested by using AI technology to match the two sides according to their preferences. Other characteristics including 'stable matching' 'Strategy proof' and 'Optimality' together make the system an ideal platform. By constructing such a platform, babysitter and households find it's more easier to find a matching without unreliable mediator while the economy benefits from more birthing and larger population in the long run.

\section{REFERENCES}

[1] Chen, G. New characteristics and trends of population development in China, 2021, DOI: People.cn.

[2] 58.com Report on Employment and Consumption in China's Care Market, Beijing Business Today, 2019.

[3] Ren, C. Research on the Development Dilemma of Community Housekeeping Service Enterprises, Modern Communication, 2015.
[4] Gale, D., \& Shapley, L. S. College Admissions and the Stability of Marriage, The American Mathematical Monthly, 1962, pp. 9-15.

[5] Teo, C.-P., Sethuraman, J., \& Tan, W.-P. GaleShapley Stable Marriage Problem Revisited: Strategic Issues and Applications, Management Science, 2001, pp. 1252-1267.

[6] Roth, A. E. The Art of Designing Markets, Harvard Business Review, 2007, pp: 118-126.

[7] Roth, A. E. The Economics of Matching: Stability and Incentives, Mathematics of Operations Research, 1982, pp. 617-628.

[8] Gale, D., \& Sotomayor, M. Some Remarks on the Stable Matching Problem, Discrete Applied Mathematics, 1985, pp. 223-232.

[9] Huo Xiangbo. How to break the chaos in the housekeeping service industry? Hebei Daily, 2021.

[10] Ling, Y., Song, Z., Yu, Y., \& Jiang, T. Dealing with an aging China -- Delaying retirement or the second-child policy? Public Library of Science, 2021, pp. 1-20. 